\title{
THE CASE FOR THE PHILOSOPHY OF CHEMISTRY
}

\begin{abstract}
The philosophy of chemistry has been sadly neglected by most contempory literature in the philosophy of science. This paper argues that this neglect has been unfortunate and that there is much to be learned from paying greater philosophical attention to the set of issues defined by the philosophy of chemistry. The potential contribution of this field to such current topics as reduction, laws, explanation, and supervenience is explored, as are possible applications of insights gained by such study to the philosophy of mind and the philosophy of social science.
\end{abstract}

\section{INTRODUCTION}

Even a cursory review of contemporary literature in the philosophy of science reveals an interesting fact: while there are separate sub-disciplines for "the philosophy of physics" and "the philosophy of biology", there is one foundational science that is missing. Why has there been no philosophy of chemistry?

Of course there has been a small literature dealing with some special philosophical problems in chemistry, sometimes even yielding an article with "philosophy of chemistry" in its title. Indeed, recently the field has seen both a special issue of Synthese $e^{1}$ (1986) devoted to a symposium on "The Philosophy of Chemistry", and a symposium held at the biennial meeting of the Philosophy of Science Association (1994) on "The Philosophy of Chemistry". ${ }^{2}$

But while the quality of the literature that has appeared so far has been very high, the quantity is out of all proportion to that of the philosophy of physics and the philosophy of biology, and what has appeared has not yet convinced most philosophers of science of the legitimacy of chemistry as an area of philosophical concern. That is, even if one wishes to argue the point of whether there does already exist a small sub-discipline of the philosophy of science called "The Philosophy of Chemistry", even its practitioners must admit that it is still in its infancy, or at least that it is "preparadigmatic". And, perhaps most obvious, the philosophical literature on any aspect of chemistry is extremely sparse, especially when considering that physics, chemistry, and biology are the dominant triumvirate in the 
natural sciences. One must conclude that the set of issues one would naturally associate with a "Philosophy of Chemistry" has at least been radically ignored.

But why? Is it that there is nothing interesting for philosophers to say about chemistry as a scientific discipline? Is chemistry in and of itself perhaps less fruitful as a source of interest for the traditional concerns of philosophers of science? Or is it, more likely, the prejudice that given chemistry's unique relationship to physics, every interesting philosophical issue that might arise as a result of studying chemistry is merely an artifact of some more interesting on-going area of concern within the philosophy of physics? $?^{3}$ That is, just as it is widely believed that chemistry can be perfectly reduced to physics, so perhaps it is believed that the philosophy of chemistry can be perfectly reduced to the philosophy of physics.

But even if one did happen to believe that all of the interesting facts about chemistry as a philosophical subject were eclipsed by the concerns of the philosophy of physics, such a position would need to be motivated. That is, one would need to feel confident that the sources of possible philosophical concern within chemistry had at least been identified by philosophers of physics, then to show that they could be happily abandoned as a result of the foundational level at which such issues were already being dealt with by philosophers of physics. One does not, however, gain such confidence in looking at contemporary literature in the philosophy of science. Indeed, far from looking to other fields like chemistry for philosophical fodder, until the relatively recent appearance of the philosophy of biology, the philosophy of science itself was dominated by the philosophy of physics, which in turns appears to be practically exhausted by quantum mechanics, relativity, and space-time (Hull 1979, Cartwright 1979).

Given the unique placement of chemistry between physics and biology in the traditional hierarchy of the natural sciences, however, isn't it reasonable to assume that chemistry may yield a set of issues worthy of increased philosophical attention? Indeed, one might point out that chemistry has traditionally been, and continues to be, the science concerned with the nature of the elements, of substance and indeed of the nature of matter, again all traditional philosophical questions. We should not be too misled by the fact that the study of matter, during the twentieth century, seems to have slipped out of the hands of chemists and into those of theoretical physicists. Falling into such a trap would be doubly erroneous, since physics has only usurped chemistry when it comes to the micro-structure of matter and secondly because it would be question begging over the issue of the reduction of chemistry, which we claim is the one of the main areas in which philosophical interest in chemistry should be directed. And, 
even if one is convinced that chemistry ultimately is reducible to physics, chemistry itself has not withered away. Why so, then, the philosophy of chemistry?

In this paper, we will argue that the Philosophy of Chemistry is an important area of study within the philosophy of science in its own right, and we will seek to identify some chemical issues which we believe worthy of increased philosophical attention. Moreover, we will argue that the insights gained through studying the philosophy of chemistry may pay dividends for more traditional debates within the philosophy of the special sciences. At the end of this volume, we have also offered a comprehensive bibliography of works that have appeared so far, which might be grouped under the heading "Philosophy of Chemistry", in the hope that philosophical debate will be furthered by knowing what has already been done.

\section{REDUCTIONISM}

Perhaps the most appropriate place to begin our analysis is with the issue of reductionism, due to the unique ontological relationship that exists between chemistry and physics. Indeed, it is the closeness of this relationship that has probably led many philosophers of science to assume that the reduction of chemistry to physics is both trivial and inevitable. But does chemistry provide such a paradigm case for reductionism? And, if so, why have so many chemists (and even physicists) been reluctant to eclipse the concerns of chemistry with those of physics? Or, does the relationship between chemistry and physics instead highlight a case where despite ontological dependency, we wish to preserve the epistemological and explanatory autonomy of our original subject?

Of course, we must here begin by stating what we mean by the blanket term "reduction", and what we take to be some of the problems it faces. ${ }^{4}$ First of all, we will not be primarily concerned with the ontological dependence of chemistry upon physics. As stated, we believe the ontological dependence of chemistry on physics to be almost a foregone conclusion. Rather, our concern will be with the epistemological reduction of chemistry to physics - with the question of whether our current description of chemistry can be reduced to our most fundamental current description of physics, namely quantum mechanics - and with its explanatory consequences. $^{5}$

The debate over reduction has had a long and storied history within the philosophy of science, and there is continuing debate over the adequacy of different accounts of it. ${ }^{6}$ The classic, and still widely embraced, view of reductionism has been given by Ernest Nagel, in his book The Struc- 
ture of Science. ${ }^{7}$ Nagel-form reduction involves the axiomatization of both theories in question and an examination of whether certain formal relationships exist between the axiomatized versions of these theories. First of all, it is not clear that the laws of chemistry, if indeed they exist, (a topic to be discussed later) can be axiomatized. Secondly, even in cases where axiomatization of the two theories at issue has been affected it is not clear that the formal condition for a successful reduction have ever been met successfully. In other words, it is not clear whether any reductions at all of the Nagel form have ever been identified. However, these facts have not prevented some philosophers from simply asserting that chemistry does reduce to physics (Kemeney and Oppenheim 1956).

In addition to Nagel's account, there exist a plethora of other ways in which the term reduction has been discussed in the philosophical literature. Rather than here rehearsing these discussions on what one might mean by reduction, we now wish to concentrate on a form of reduction which has been discussed previously by one of us (Scerri 1994). We claim that this may be one of the ways in which thinking about chemistry may help to clarify issues in the philosophy of science and henceforth we shall focus on a more naturalistic approach to reduction, which may be described under the label of quantitative reduction.

Now, what might a chemist say about the reduction of chemistry? If one were to ask a contemporary chemist whether chemistry could be reduced to physics, he or she would probably direct us to a colleague in computational quantum chemistry, since these are the specialists who deal with such issues. A cursory examination of this branch of theoretical chemistry shows it to be an attempt to calculate the properties of atoms and molecules (including their reactivities) from first principles. This task is carried out via the Schrödinger equation, which may be described as the main workhorse of applied quantum mechanics. It should be said that there are many other pursuits in theoretical and physical chemistry which also broadly speaking fall under the umbrella of attempts to reduce chemistry. These other areas would include so called semi-empirical calculations in which certain experimental data are "fed in by hand" as it were. In such cases the philosopher will immediately object (with full justification) that such an approach, even if it were successful, would not constitute a genuine reduction, since one would be using not merely the reducing theory of quantum mechanics, but also some ingredients which belong to the science to be reduced, namely chemical data.

Thus, in order to be as even handed as possible, and to give the supposed reduction of chemistry the best chance of success, one would need to examine the research in the area of ab initio calculations, in which no 
experimental data whatsoever are admitted into the computations. ${ }^{8}$ The aim here is simply to calculate the energy of a molecule, a bond angle, a dipole moment, or a rate of reaction from the first principles of quantum mechanics. ${ }^{9}$ How does this enterprise fare in contemporary chemistry?

We believe that even given this rather extreme perspective on the reduction of chemistry, the situation is one of an incomplete reduction. In one sense the failure of this reduction is very easily realized once one considers that the application of the Schrödinger equation even to a system as simple as the helium atom lands us directly at the door of the many-body problem. Solutions to the many-body problem are necessarily approximate, as is well known in physics. The sad fact for chemistry, moreover, is that only the Schrödinger equation for the hydrogen atom possesses an exact solution. This system is hardly of interest to "real chemists", whose concerns range over the remaining hundred or so elements in the periodic table. And even if one were to restrict attention to the element hydrogen, chemists are more frequently concerned with diatomic hydrogen $\left(\mathrm{H}_{2}\right)$ rather than the highly reactive hydrogen atom which is the only system to have been truly captured by quantum mechanics.

Nonetheless, leaving aside the inevitability of approximations in chemistry, we might still want to inquire as to how good the approximations actually are. This question should be approached rather carefully, and we suggest that a critical attitude should be adopted towards the claims made by the practitioners in the field. The source of latitude exercised by computational quantum chemists lies in a particular technical aspect which underlies much of the computational work. The approximations used in computational quantum chemistry involve the expansion of wavefunctions as infinite series of terms in much the same way as Fourier analysis seeks to represent a complex function as an infinite series of separate terms. The simple fact of the matter is that one may model a complex function to virtually any degree of precision, provided one is prepared to introduce an increasing number of correction terms in the series expansion. By adding greater flexibility to the wavefunction, something which can always be justified on a post hoc basis, one can obtain increasingly better approximations to the experimentally observed data which one is trying to calculate.

Admittedly, there is considerable virtue in demonstrating that a particular calculation works well in a certain test case, and then adopting the same approach to an unknown experimental situation. However, such an approach, referred to as the "calibrated ab initio method" may be justly criticized by the purist on much the same grounds as we discussed earlier in the case of semi-empirical calculations. ${ }^{10}$ Both procedures involve the 
importation of data from the level of facts to be reduced into the reducing theory.

There are measures which can be taken to estimate the goodness of the calculation independently of the experimental data and which thus lend the calculation greater reliability, but these are notoriously difficult procedures, and many computational quantum chemists become irritated at the very mention of such internal procedural checks. ${ }^{11}$

Essentially, these internal estimates consist in the determination of both upper and lower bounds in the calculation of any particular property such as the energy of a molecule. The problem arises because whereas the variation method, which lies at the heart of most approximations, can provide an estimate of the upper bound to the energy, the systematic calculation of the lower bound is still an open problem in quantum chemistry. ${ }^{12}$

Thus, we see from our example of the explanation, or more correctly the calculation, of chemical facts from first principles, that there are many difficulties for the reductionist view, which face the computational chemist. Of course, chemistry is concerned with a far broader range of explanations than those exhausted simply by calculating a certain property such as a dipole moment or a bond angle. To rest contented with only this notion of reduction in chemistry would surely be to adopt a rather narrow stance on what constitutes epistemological reduction. Still, the difficulties of the kind mentioned above reveal the weakness of an explanatory approach which overestimates the ease with which chemical phenomena can be perfectly captured and explained by theoretical notions drawn from physics.

We have concluded that quantitative reduction, the most overt attempt at reduction in chemistry, has not been achieved. However, we also note that the present failure to obtain simultaneous upper and lower bounds to $a b$ initio calculations may eventually be overcome. If this problem were ever to be solved it would be possible to speak of approximate reduction of quantitative properties which are important in chemistry, such as the energies of molecules or bond angles. Full reduction would nevertheless remain unattainable since, as mentioned above, the Schrödinger equation only possesses an exact solution in the case of the hydrogen atom..$^{13}$

However, we wish to carefully distinguish between the above form of "quantitative reduction"14 of chemistry and what might be termed "conceptual reduction". ${ }^{15}$ Such a distinction already has been made in the philosophy of chemistry, though perhaps somewhat obliquely, by Hans Primas, the author of the only book on the reduction of chemistry:

Many calculations have been extremely sophisticated, designed by some of the foremost researchers in this field to extract the maximum amount of insight from quantum theory. For simple molecules, outstanding agreement between calculated and measured data has been obtained. Yet, the concept of a chemical bond could not be found anywhere in these 
calculations. We can calculate bonding energies without ever knowing what a bond is! (Primas 1983).

It is conceptual reduction which is of greater relevance for the remainder of the present article. By conceptual reduction we mean attempts to reduce chemical concepts such as composition, bonding, and molecular structure. ${ }^{16}$ In these cases we are not obliged to adopt an agnostic approach due to the present state of science. Rather, our claim is that this form of reduction is not even possible in principle due to the very nature of the concepts themselves. That is, the concepts of composition, bonding, and molecular structure cannot be expressed except at the chemical level.

Mario Bunge, for example, has made the point that the concept of chemical composition cannot be reduced to physics. Bunge writes,

At first sight chemistry is included in physics because chemical systems would seem to constitute a special class of physical systems. But this impression is mistaken, for what is physical about a chemical systems is its components rather than the system itself, which possesses emergent (though explainable) properties in addition to physical properties. (Bunge 1982)

Bunge cites as an example of such an emergent property that of having a composition that changes lawfully in the course of time. The atomic and molecular components do not show this property of composition. Primas, as quoted earlier, says that we can calculate certain molecular properties, but we cannot point to something in the mathematical expressions which can be identified with bonding. The concept of chemical bonding seems to be lost in the process of reduction (Primas 1983).

Woolley, to cite another example, has raised a great deal of interest in saying that chemical structure cannot be found in the pure quantum mechanical formalism applied to a chemical system. Structure, he tells us, is imposed by using the Born-Oppenheimer approximation (Woolley 1978). One can do calculations which do not draw on the Born-Oppenheimer approximation, and not only do we obtain solutions but in some cases, such as molecular beam experiments, we can do more accurate calculations by omitting the Born-Oppenheimer approximation altogether. According to Woolley, the concept of molecular structure is absent at the quantum mechanical level. ${ }^{17}$

In each of the cases cited, as well as others, we encounter chemical concepts that seem irreducible. Thus, even where chemical relationships may at base be dependent upon physical ones, it nonetheless seems perfectly appropriate to reject reductive explanations in some instances, where the concepts we employ and even the very explanandum itself may be lost in the theoretical terms of the primary science. That is, we may admit 
the ontological dependence of chemical facts upon physical ones, and yet eschew the epistemological reduction of chemistry to physics.

\section{EXPLANATION}

Another potential area of interest in the philosophy of chemistry, closely related to the concerns of reductionism, would seem to lie in the investigation into the nature of chemical explanations. What do explanations in contemporary chemistry consist in? Are they "autonomous" from the concerns of physics? In what way?

At first brush, one may think that the close ontological relationship between chemistry and physics would inevitably bias chemical explanation in favor of reductionism. We shall see, however, that there is good reason to support the autonomy of chemical explanations. That is, even if we admit that chemical regularities are instantiated in physical relationships, it may be most useful, perhaps, to describe and explain them at the chemical (secondary) level of inquiry.

One very important form of explanation which pervades all areas of chemistry, from teaching to frontier research, lies in talk of electron shells or orbitals, as they are often called. The formation of bonds, acid-base behavior, redox chemistry, photochemistry, reactivity studies, etc., are all regularly discussed by reference to the interchange of electrons between various kinds of orbitals.

This approach may at first sight seem to speak in favor of the epistemological reduction of chemistry to physics, since talk of electron shells is thought to belong primarily to the level of atomic physics. However, a more critical examination of the issues involved reveals no such underpinning from fundamental physics. It emerges that explanations in terms of electron orbitals, and indeed all talk of orbitals in chemistry, is not sanctioned by our present understanding of quantum mechanics. The remarkable fact is that at the most fundamental quantum mechanical level electronic orbitals become ontologically redundant. Electronic orbitals simply do not exist according to quantum mechanics, although they remain as a very useful explanatory device. This result is embodied in the more fundamental version of the Pauli Exclusion Principle, which is frequently forgotten at the expense of the restricted and strictly invalid version of the Principle, which does uphold the notion of electronic orbitals (Scerri 1991, 1995).

This situation implies that most explanations given in chemistry which rely on the existence of electrons in particular orbitals are in fact "level specific" explanations, which cannot be reduced to or underwritten by quantum mechanics. ${ }^{18}$ Thus, a case has been demonstrated where the 
explanation of what it is that we seek to know when we engage in chemical explanation would seem to suggest that we eschew reductive explanations, and support the explanatory autonomy of chemistry.

\section{LAWS}

Just as there are some typically chemical explanations, such as talk of electron orbitals, which are best pursued at an autonomous level of explanation, we now suggest that there are also some irreducibly chemical laws as well. A good example of such a chemical law is provided by the so called periodic law, as first discovered by Mendeleev and Meyer, but anticipated by many others. ${ }^{19}$ Viewed from the perspective of physics, the status of the periodic system may appear to be far from law-like. Significantly, the periodic law seems not to be exact in the same sense as are laws of physics, for instance Newton's laws of motion. Loosely expressed, the periodic law states that there exists a periodicity in the properties of the elements governed by certain intervals within their sequence arranged according to their atomic numbers. ${ }^{20}$ The crucial feature which distinguishes this form of periodicity from that found in physics is that chemical periodicity is approximate. For example, the elements sodium and potassium represent a repetition of the element lithium, which lies at the head of group I of the periodic table, but these three elements are in no sense identical. Indeed, a vast amount of chemical knowledge is gathered by studying patterns of variation which occur within vertical columns or groups in the periodic table.

Predictions which are made from the so called "periodic law" do not follow deductively from a theory in the same way in which idealized predictions flow almost inevitably from physical laws, together with the assumption of certain initial conditions. So, can the case be made that the periodic law should legitimately be considered a law of chemistry, and, moreover, that it is explanatorily irreducible? We shall argue that it can.

The historical facts surrounding the classic predictions of a number of unknown elements made by Mendeleev show that he used a vast store of chemical intuition, rather than a straightforward algorithm as a physicist might, when operating with a physical law. A clearer appreciation of the nature of the periodic "law" can be gained by considering how Mendeleev arrived at the specific details of his predictions on the elements gallium, germanium and scandium. Mendeleev himself gives a clear and unambiguous indication of his method in his textbook The Principles of Chemistry. The method consists of simultaneous interpolation within groups or columns as well as within periods or rows of the periodic table. This procedure is 
achieved very simply by taking the average of the sum of the values of the four elements flanking the element in question. According to Mendeleev,

If in a certain group there occur elements, $R_{1}, R_{2}, R_{3}$, and if in that series which contains one of the elements, for instance $R_{2}$, an element $Q_{2}$, precedes it and an element $T_{2}$ succeeds it, then the properties of $R_{2}$ are determined by the mean of the properties of $R_{1}, R_{3}, Q_{2}$ and $T_{2}$. (Mendeleev 1905)

In the various editions of his textbook, and in the publications dealing specifically with his predictions, Mendeleev repeatedly gives the example of calculating the atomic weight of the element selenium, a property which was known at the time and which could thus be used to test the reliability of his method.

However, if one attempts to apply this method to the prediction of atomic weights, atomic volumes, densities and other properties of gallium, germanium and scandium one arrives at values which differ significantly from Mendeleev's published predictions. It should also be noted that Mendeleev's published predictions were generally remarkably accurate when compared with the properties of the subsequently discovered elements. This suggests great chemical intuition on the part of Mendeleev which allowed him to make minor modifications from his stated method whenever it was necessary. Mendeleev appears to be deviating from his professed approach, but nowhere does he deem it necessary to specify how and why he departs from the simple method of interpolation. This is one of the many examples which illustrate the approximate nature of his periodic law.

Nevertheless, Mendeleev considered the periodic law as sufficiently law-like in that it could not tolerate any exceptions, such as any deviation from the ordering of the elements according to atomic weight. In the case of the elements tellurium and iodine, for example, he predicted that the atomic weights of these two elements were in error since the available values suggested the opposite order to the one dictated by chemical properties. More specifically, tellurium showed a higher atomic weight according to the then measured values and an ordering of the elements based on this feature would have placed tellurium in the same chemical group as fluorine, chlorine, and bromine, where it did not belong in chemical terms. As it turned out, Mendeleev was correct to reverse the ordering but for the wrong reason. The atomic weights had in fact been approximately correct, but the ordering of the elements is better achieved by means of the atomic number of each element. This improved ordering scheme resulted from the work of Moseley in 1912, and its virtue lies in overriding any complications due to the isotopic mixture which occurs in most chemical elements. 
Mendeleev had sufficient confidence in his periodic law to use it to predict the existence of several new elements, and the properties of their compounds, in addition to correcting the atomic weights of some already known elements. Nevertheless, this predictive aspect seems to have been overemphasized by historians of chemistry and writers of chemistry books. It appears that Mendeleev's ability to accommodate the already known elements may have contributed as much to the acceptance of the periodic system as did his dramatic predictions. For example, the citation which accompanies his being awarded the Davy Medal by the Royal Society of London makes no mention whatsoever of his predictions ${ }^{21}$ (Scerri 1996).

Indeed, the major reason why it is Mendeleev above all others who is credited with discovering chemical periodicity is that he elevated the periodic law to the status of a law of nature, and spent the remainder of his life in boldly examining its consequences and defending its validity. Nor was this a simple task since the periodic system was frequently challenged by subsequent discoveries of new elements. For example, in 1913 Ramsey and Rayleigh discovered the element argon, followed by a number of other noble gases. The name of these elements derives from their extreme reluctance to enter into chemical combination with other elements, a fact which led some chemists to suggest that they did not even belong in the periodic table. The noble gas elements had not been predicted by Mendeleev or anybody else and it required five years of intense effort by chemists and physicists before they were eventually successfully accommodated into the table. This was done in the form of a completely new column situated between the halogens and the alkali metals.

To sum up, we have argued that the periodic law is regarded as highly law-like in chemistry and that there exist no exceptions to it. Yet, the nature of the law is such that it cannot be captured by a simple numerical relationship, ${ }^{22}$ and the regularity it captures cannot be expressed in nomological fashion using non-chemical concepts. The law expresses an approximate trend among the properties of the elements and their compounds. But if one attempts to express this trend numerically, such a relationship is found to hold only approximately. The periodic law thus stands as an autonomous law of chemistry. We disagree with Hettema and Kuipers who claim that the periodic table has been reduced to "atomic theory" 23 (Hettema and Kuipers 1988). This erroneous claim is based on the fact that elements within any one group tend to share the same outer shell configuration. However there are numerous exceptions to this model and the possession of a particular configuration is neither necessary nor sufficient for inclusion of an element in any particular group of the table. The reduction of the periodic table should in our view mean the ability to 
calculate exactly the total energies or other properties of the atoms in the periodic table. Such a reduction is at best approximate as it is subject to the usual limitations of solving the many-body Schrödinger equation.

\section{SUPERVENIENCE}

The notion of supervenience has been much discussed in recent years throughout the philosophy of science. ${ }^{24}$ Indeed, some authors have even drawn on the relationship between chemistry and physics to illustrate their basic arguments about the supervenience relationship (Papineau 1993). In doing so, however, it has been assumed that the relationship between chemistry and physics does not raise any particular problems of its own. Thus, as in many discussions of the appropriateness of chemistry and physics as an example of pure reduction, the relationship between chemistry and physics has been taken by some to represent a paradigmatic case of true supervenience. But is this move warranted?

Although there is notoriously no unanimous agreement on what the supervenience relationship is, the most popular view is that supervenience is a relationship of asymmetric dependence. Two macroscopic systems which have been constructed from identical microscopic components are assumed to show identical macroscopic properties, whereas the observation of identical macroscopic properties in any two systems need not necessarily imply identity at the microscopic level. In simpler terms, the phenomena we study in some secondary science are thought to be ontologically dependent upon relationships at the primary level. This argument has been widely used throughout the philosophy of science (and the philosophy of mind) as a rescuing maneuver from the impasse produced by the failure to establish the epistemological reducibility of any of the special sciences. Why? For the simple reason that supervenience is taken to make no guarantee about the epistemological or explanatory consequences which would necessarily follow from even a strong ontological dependence between two different descriptive levels. ${ }^{25}$ Thus, the claim has been that supervenience allows us the virtue of ontological dependence, without the vice of explanatory reduction - it allows us to "have our cake and eat it too", as the saying goes.

Thus, although reductionism may fail in the traditional sense (that is, although there may be a breakdown in our efforts to establish a seamless continuity between the special sciences and quantum mechanics), we yet can maintain that, deep down, chemical or biological systems are governed solely by physical laws. Materialism is rescued, even though reduction has been found to flounder. Moreover, we need not appeal to "ontological 
emergence", the "supernatural", or other questionable notions in order to get our epistemological autonomy.

Some authors, however, have suggested that this attempt to rescue explanatory autonomy from the jaws of ontological dependence is nothing more than a sleight of hand. As Paul Teller asks in the title of his paper, "Is Supervenience Just Disguised Reduction?"26 Leaving aside the fascinating questions raised by this more general debate, we believe it is here interesting to examine the appropriateness of the use of the relationship between chemistry and physics to demonstrate the supervenience relationship.

\section{DOES CHEMISTRY SUPERVENE ON PHYSICS?}

In considering an answer to our above question, we shall consider the property of "smell" in order to examine more specifically what the supervenience of chemistry on physics might entail. According to the general supervenience argument which was outlined above, we take it that if two chemical compounds were "constructed" out of elementary particles in an identical manner, they would share the same smell (among their macroscopic properties). This is a philosophical claim which could be examined with respect to what is known empirically about the chemistry of smell. Similarly, the supervenience argument would entail that if two compounds share the same macroscopic property of smell, we could not necessarily infer that the microscopic components from which the compounds are formed would be identical. In the following, we do not propose to give an analysis of the state of the art in the chemistry of smell, but will restrict ourselves to a few general philosophical remarks, which highlight the relevance of considering such an issue as a legitimate one for the Philosophy of Chemistry.

It would appear that two opposing possibilities present themselves:

(A) Why is it that we are prepared to accept the notion that two compounds which have the same smell do not have an identical microstructure? Could it be because the property of smell is a rather vague concept, which does not seem susceptible to quantification? However, smell is indeed an explanatory concept in chemistry, and in keeping with our previous remarks about chemical explanations, such explanations cannot be reduced without remainder to physics, as compared to some other chemical data which do seem susceptible to approximate reduction.

On the other hand, if smell were rendered quantitative by some future advances, then we might be justified in insisting that two substances sharing the same numerical data regarding their degree of smell should share exactly the same microstructure. In other words, we would expect a reduction of 
the smell-like data just as it is reasonable to expect an approximate reduction of the energy of a molecule. Were it possible to reduce these aspects of the chemistry of smell, then the claimed asymmetry in the supervenience argument would have been falsified in this case.

(B) The following intuition about the property of smell would seem to lead to the opposite conclusion, in that one would want to uphold the general spirit of what it means for smell to supervene over the physical make-up of a compound, and more specifically to uphold the claimed asymmetry.

Smell is a rather curious property in that its perception is thought to arise from a lock-and-key mechanism, whereby a certain molecular shape will trigger a particular smell receptor, and thus produce the sensation of a particular odor. Seen in this way, it would seem that two vastly different molecules which share the same molecular side-chain (which is required to trigger a certain smell receptor) would do so irrespective of the structure of the rest of the molecule. This view would suggest that the same smell might indeed result from different molecules having different microscopic components

Such questions could be answered more definitively by biochemists and neurophysiologists. But whatever the outcome of this case, the question of the supervenience of chemistry on physics would seem to depend precisely on the empirical facts, and the conclusions which they support, that are to be drawn from cases like the one considered above, and not from more general philosophical musing about chemistry and physics. Indeed, if the alleged supervenience of chemistry on physics is meant to provide an exemplar for the supervenience relationship (and as such a stringent test case for the more far-reaching question of the supervenience of biology on physics, or mental states on physical states) more work within what may now be thought of as the Philosophy of Chemistry seems in order.

Thus, we hope by now to have established the following two conclusions: (1) that there should be more scholarship within "The Philosophy of Chemistry" in its own right, given the unique light which it sheds on some of the most important debates within the philosophy of science, and (2) that there is much to be learned in the application of our conclusions from the case of chemistry to other debates within the "special sciences". In the next section, we shall briefly consider a few such issues, which the Philosophy of Chemistry raises for the Philosophy of Mind, and the Philosophy of Social Science. 


\section{LESSONS FOR THE SPECIAL SCIENCES}

We shall now briefly consider the importance of the issues which the Philosophy of Chemistry has raised for such topics as reductionism, explanation, laws, and supervenience, in the application to existing debates within the Philosophy of Mind and the Philosophy of Social Science. What can the special sciences learn from the case of chemistry?

Perhaps the greatest source of interest which the philosopher of mind or the philosopher of social science may have in the philosophy of chemistry is simply to learn that, from a certain point of view, chemistry too can be considered a "special science". And attendant upon this is the realization that what previously may have been considered a relatively uncontroversial case of ontological dependence raises the very same issues regarding reducibility, the autonomy of explanations (nomological and otherwise) at secondary levels, and the appropriateness of supervenience, that have long dogged many of the traditional debates within the philosophy of mind and the philosophy of social science.

Far from suggesting, however, that chemistry provides a paradigm case of conceptual emergence, or unequivocal support for the autonomy of lawlike relationships in a secondary science, our point here is merely that those who are interested in debates about the dependence of the mental upon the physical, the appropriateness or possibility of social scientific laws, and the implications of supervenience, have a lot to learn from chemistry. Here, after all, is a science whose ontological dependence upon physics is not in doubt. Chemistry suffers from none of the worries about "vital forces", "consciousness", or "intentionality" which have bedeviled the debate about laws in developmental and evolutionary biology or the social sciences. Yet, even given the purely material nature of the relationship between the subject matters of chemistry and physics, we note that many issues - like supervenience or the autonomy of law-like explanations - can here be studied in their purest form. Does strong ontological dependence not only allow epistemological autonomy, but also suggest that in some cases nonreductive explanations are preferable? If causal forces are discernible at the primary level, does this suggest that irreducible nomological explanations cannot also be rendered at the secondary level? How valuable is the concept of supervenience in allowing us to maintain material dependency while also supporting irreducibility?

Such questions can perhaps be analyzed in their purest form within the philosophy of chemistry, then to be applied back to debates within the special sciences. For instance the special scientist might ask: Does the sort of conceptual irreducibility we have supported within the philosophy of chemistry easily transfer to debates within the philosophy of mind? 
Does the suggestion that one can favor autonomous nomological explanations within chemistry, while still recognizing the fundamental ontological dependence of chemical relationships upon physical ones, suggest that there can be laws in the social sciences? ${ }^{27}$ Such questions represent an important source of interest which other philosophers may have in the philosophy of chemistry. No longer satisfied merely to draw examples from chemistry, to support whatever conclusion is desired in debates about the secondary sciences, we are here advocating that many of the concepts themselves have a richness which can be appreciated only by considering the facts of the unique relationship of chemistry to physics.

\section{CONCLUSION}

In this essay, we have offered several arguments in support of greater philosophical attention to what may now be called "The Philosophy of Chemistry". Such a field may pay rich dividends, not only for philosophical study about chemical topics in their own right, but also for the importance of the conclusions one might draw from this field for application to existing debates throughout the philosophy of science. We have here provided several suggestions for further work, and have appended a comprehensive bibliography of works that have to date appeared within the philosophy of chemistry, in order to allow such study now to go forward.

It has of late become a truism within the philosophy of science that $a$ priori solutions to philosophical matters concerning the sciences are stale without attention to empirical details drawn from the sciences themselves. A corollary view might now also be offered: that what many have dismissed as straightforwardly empirical matters concerning the relationship between the sciences may also benefit from renewed philosophical attention.

\section{NOTES}

* The authors would like to thank James Woodward, Kim Sterelny and Jeff Ramsey for their comments on an earlier version of this article.

${ }^{1}$ Vol. 69, No. 3 (December 1986).

${ }^{2}$ Published in PSA 1994, Vol. 1 (East Lansing, Mich.: Philosophy of Science Association, 1994).

${ }^{3}$ Even some of the studies purported to deal with the reduction of chemistry are in effect disguised studies into the foundations of quantum mechanics. In our view this is especially true in the work of Primas (1983).

${ }^{4}$ Despite the ubiquity of the term "reduction" in the philosophy of science, there is a fierce debate over how it should be defined. The classic definition can be found in Ernest Nagel, The Structure of Science (New York: Harcourt, Brace, and World, 1961). Perhaps 
the most comprehensive analysis of the different possible senses of the term "reduction" can be found in Lawrence Sklar, 'Types of Inter-Theoretic Reduction', British Journal for the Philosophy of Science, Vol. 18 (1967), pp. 109-124. In the present paper we shall be interpreting reduction in the more traditional sense, as an epistemological relationship between scientific theories that bears directly on the issue of scientific explanation. It is our opinion, therefore, that talk of "ontological reduction" only confuses the issue. Cf. F. Ayala (1974) in Studies in Philosophy of Biology, F. Ayala and T. Dobzhansky (eds.), (Berkeley: University of California Press).

${ }^{5}$ We make no apologies for taking such an unavoidably piecemeal approach, which we understand will not be to the taste of some philosophers from the "old school" who believe that one should only deal in generalities and who might instinctively wish to avoid grappling with the sordid details of present day chemistry and physics.

${ }^{6}$ For an introduction to the literature on this debate, see the bibliography in Sklar, "InterTheoretic Reduction", cited in Note 4 above.

${ }^{7}$ New York: Harcourt, Brace, and World, 1961.

${ }^{8}$ The only experimental values admitted into genuine $a b$ initio work are those of fundamental constants such as the mass and charge of the electron.

${ }^{9}$ A recent review of the work in ab initio quantum chemistry can be found in M. HeadGordon, 'Quantum Chemistry and Molecular Processes', Journal of Physical Chemistry 100, 13213-13225 (1996).

${ }^{10}$ Most practitioners of quantum chemistry do not consider this a serious criticism and continue to label the methods as ab initio although they are strictly speaking not so in the sense which we intend.

${ }^{11}$ Handy, N., (1992), 'Pople and Boys', Chemistry in Britain 28, 709-709.

12 Weinhold, F., (1972), 'Upper and Lower Bounds to Quantum Mechanical Properties', Advances in Quantum Chemistry, Vol. 6, 299-331.

${ }^{13}$ Even here one should perhaps remain agnostic regarding reduction, since it is not inconceivable that quantum mechanics will be replaced by a theory which admits exact solutions even in chemically interesting cases.

${ }^{14}$ One of us (Eric Scerri) has previously referred to this form of reduction as "pragmatic reduction". Scerri, E. R., 'Has Chemistry Been At Least Approximately Reduced to Quantum Mechanics?', PSA 1994, Vol. 1 (East Lansing, Mich.: Philosophy of Science Association), 160-170.

${ }^{15}$ Previous failure to draw such a distinction has resulted in much confusion regarding the status of claims about the reduction of chemistry.

${ }^{16}$ Some of these issues are discussed in the article by J. van Brakel in this issue.

${ }^{17}$ This claim is controversial and is disputed in the accompanying article by Ramsey, among others.

${ }^{18}$ Curiously, the opposite conclusion seems to have been reached in the field of chemical education. The fact that chemical explanations frequently appeal to electronic orbitals is wrongly taken as a reason for basing the presentation of chemistry on quantum mechanics. This is to commit the "orbital fallacy". E. R. Scerri (1991), 'Chemistry, Spectroscopy and the Question of Reduction', Journal of Chemical Education, Vol. 68, 122-126.

${ }^{19}$ Scerri, E. R., 'The Evolution of the Periodic Table', Scientific American (forthcoming).

${ }^{20}$ The length of the intervals between recurring elements varies throughout the periodic table being $2,8,8,18,18,32$, etc.

${ }^{21}$ The question of whether Mendeleev triumphed because of making predictions rather than for accommodating the properties of the elements known at the time has been the source of much discussion among philosophers of science interested in the debate regarding pre- 
diction and accommodation of data by scientific theories. Several examples are: Brush, S. J. (1989) 'Prediction and Theory Evaluation', Science Vol. 246, 1124-1129; Gardner, M. R. (1982) 'Predicting Novel Facts”, British Journal for the Philosopy of Science, Vol. 33, 1-15; Lipton, P. (1990) 'Prediction and Prejudice', International Studies in the Philosophy of Science, Vol. 4, 51-65; Maher, P. (1988) 'Prediction, Accommodation and the Logic of Discovery', PSA 1988, Vol. 1 (East Lansing, Mich.; Philosophy of Science Association), 273-285. The appropriate evaluation of the role of the periodic table regarding prediction and accommodation may be one example of the relevance of the philosophy of chemistry to work in the philosophy of science (Scerri, Worrall, in preparation).

${ }^{22}$ As Löwdin has written, "it is perhaps remarkable that, in axiomatic quantum theory, the simple energy rule (order of filling of orbitals) has not yet been derived from first principles”. P. O. Löwdin (1969), 'Some Comments on the Periodic System of the Elements', International Journal of Quantum Chemistry IIIS, 331-334. Neither has this situation changed since Löwdin wrote these words.

Recently Rouvray has re-emphasized how quantum mechanics does not provide a true reduction of the periodic table. D. H. Rouvray (1996), 'The Surprising Periodic Table: Ten Remarkable Facts', Chemical Intelligencer, July: 39-47.

${ }^{23}$ In any case it is not clear precisely what Hettema and Kuipers intend by this phrase.

${ }^{24}$ See for example, Jaegwon Kim (1984), 'Concepts of Supervenience', Philosophy and Phenomenological Research, Vol. 45(2), 153-176. An excellent collection of current papers on supervenience can be found in Horgan, T. (1983) (ed.), 'The Spindel Conference: Supervenience', The Southern Journal of Philosophy, Vol. 22, Supplement.

25 "Reduction, explanation, and the like are epistemic activities, and the mere fact that such equivalence or biconditionals 'exist' is no guarantee that they are, or will ever become, available for reductive or explanatory uses". J. Kim, 'Concepts of Supervenience', p. 173. It is interesting to note, however, that Kim no longer thinks that supervenience supports the non-reductionist program. Cf. his 'Mechanism, Purpose, and Explanatory Exclusion', in J. Tomberlin (1989) (ed.), Philosophical Perspectives, Vol. 3 (Atascadero, Calif.: Ridgeview), 77-108.

${ }^{26}$ The Southern Journal of Philosophy (1985), Vol. 23, 93-99. Teller answers this question in the negative.

${ }^{27}$ One of us (Lee McIntyre) has explored the question of the autonomy of social scientific laws in Chapter 6 of Laws and Explanation in the Social Sciences (Boulder, CO: Westview Press, 1996), where the analogy with chemistry is considered explicitly.

\section{REFERENCES}

Ayala, F.: 1974, in F. Ayala and T. Dobzhansky (eds.), Studies in Philosophy of Biology: Reduction and Related Problems, University of California Press, Berkeley.

Brush, S. J.: 1989, 'Prediction and Theory Evaluation', Science 246, 1124-1129.

Bunge, M.: 1982, 'Is Chemistry a Branch of Physics?' Zeitschrift für allgemeine Wissenschaftstheorie 13, 209-23.

Cartwright, N.: 1979, 'Philosophy of Physics', in P. Asquith and H. Kyburg Jr. (eds.), Current Research in Philosophy of Science, Philosophy of Science Association, East Lansing, MI, pp. 381-385.

Gardner, M. R.: 1982, 'Predicting Novels Facts', British Journal for the Philosophy of Science 33, 1-15.

Handy, N.: 1992, 'Pople and Boys', Chemistry in Britain 28, 709. 
Head-Gordon, M.: 1996, 'Quantum Chemistry and Molecular Process', Journal of Physical Chemistry 100, 13213-25.

Hettema, H. and T. A. F. Kuipers: 1988, 'The Periodic Table - Its Formalization, Status, and Relation to Atomic Theory', Erkenntnis 28, 387-408.

Horgan, T. (ed.): 1983, 'The Spindel Conference 1983: Supervenience', The Southern Journal of Philosophy 22, supplement.

Hull, D.: 1979, 'Philosophy of Biology', in P. Asquith and H. Kyburg, Jr. (eds.), Current Research in Philosophy of Science, Philosophy of Science Association, East Lansing, MI, pp. 421-435.

Hull, D., M. Forbes, and R. Burian (eds.): 1994, PSA 1994, Vol. 1, Philosophy of Science Association, East Lansing, MI.

Kemeney, J. G. and P. Oppenheim: 1956, 'On Reduction', Philosophical Studies VII, 6-19.

Kim, J.: 1984, 'Concepts of Supervenience', Philosophy and Phenomenological Research 45, 153-76.

Kim, J.: 1989, 'Mechanism, Purpose, and Explanatory Exclusion', in J. Tomberlin (ed.), Philosophical Perspectives, Vol. 3, Ridgeview, Atascadero, CA, pp. 77-108.

Lipton, P.: 1990, 'Prediction and Prejudice', International Studies in the Philosophy of Science 4, 51-65.

Löwdin, P. O.: 1969, 'Some Comments on the Periodic System of the Elements', International Journal of Quantum Chemistry IIIS, 331-34.

Maher, P.: 1988, 'Prediction, Accommodation and the Logic of Discovery', in PSA 1988, Vol. 1, Philosophy of East Lansing, MI, pp. 273-85.

McIntyre, L.: 1996, Laws and Explanation in the Social Sciences, Westview Press, Boulder, CO.

Mendeleev, D. I.: 1905, Principles of Chemistry, 3rd English ed., translated from 7th Russian ed. by G. Kemansky, Longmans, Green \& Co., London.

Nagel, E.: 1961, The Structure of Science, Harcourt, Brace and World, New York.

Papineau, D.: 1993, Philosophical Naturalism, Blackwell, Oxford.

Primas, H.: 1983, Chemistry, Quantum Mechanics and Reductionism, Springer, Berlin.

Rouvray, D. H.: 1996, 'The Surprising Periodic Table: Ten Remarkable Facts', Chemical Intelligencer July, 39-47.

Scerri, E. R.: 1991, 'Electronic Configurations, Quantum Mechanics and Reduction', British Journal for the Philosophy of Science 42, 309-25.

Scerri, E. R.: 1991, 'Chemistry, Spectroscopy and the Question of Reduction', Journal of Chemical Education 68, 122-26.

Scerri, E. R.: 1994, 'Has Chemistry Been at Least Approximately Reduced to Quantum Mechanics?' in D. Hull, M. Forbes, and R. Burian (eds.), PSA 1994, Vol. 1, Philosophy of Science Association, East Lansing, MI, pp. 160-70.

Scerri, E. R.: 1995, 'The Exclusion Principle, Chemistry and Hidden Variables', Synthese 102, 162-69.

Scerri, E. R.: 1996, 'Stephen Brush, The Periodic Table and the Nature of Chemistry', in P. Jannich and N. Psarros (eds.), Die Sprache der Chemie, Proceedings of the Second Erlenmeyer Colloquium on Philosophy of Chemistry, Köningshausen \& Neumann, Würtzburg, pp. 169-176.

Scerri, E. R.: 1997, 'It All Depends What You Mean by Reduction', in K. Mainzer, A. Müller, and W. Saltzer (eds.), From Simplicity to Complexity, Information, Interaction, Emergence, Proceedings of the $1994 \mathrm{ZiF}$ Meeting in Bielefeld, Vieweg-Verlag (in press).

Scerri, E. R.: forthcoming, 'The Evolution of the Periodic Table', Scientific American. 
Sklar, L.: 1967, 'Types of Inter-Theoretic Reduction', British Journal for the Philosophy of Science 18, 109-24.

Teller, P.: 1985, 'Is Supervenience Just Disguised Reduction?', The Southern Journal of Philosophy 23, 93-9.

Weinhold, F.: 1972, 'Upper and Lower Bounds to Quantum Mechanical Properties', Advances in Quantum Chemistry 6, 299-331.

Woolley, R. G.: 1978, 'Must a Molecule Have a Shape?', Journal of the American Chemical Society 100, 1073-8.

Department of Philosophy and Religion

Colgate University

13 Oak Dr.

Hamilton, NY 13346

U.S.A.

Division of Humanities and Social Sciences, 228-77

California Institute of Technology

Pasadena, CA

U.S.A. 